\title{
Combination Vaccination With Tetanus Toxoid and Enhanced Tumor-Cell Based Vaccine Against Cervical Cancer in a Mouse Model
}

\author{
Donia Alson ${ }^{1}$, Scott C. Schuyler ${ }^{1,2,3}$, Bo-Xin Yan ${ }^{1}$, Karthika Samimuthu ${ }^{1}$ and \\ Jiantai Timothy Qiu ${ }^{4,5 *}$ \\ ${ }^{1}$ Graduate Institute of Biomedical Sciences, College of Medicine, Chang Gung University, Taoyuan, Taiwan, ${ }^{2}$ Department of \\ Biomedical Sciences, Chang Gung University, Taoyuan, Taiwan, ${ }^{3}$ Division of Head \& Neck Surgery, Department of \\ Otolaryngology, Chang Gung Memorial Hospital, Taoyuan, Taiwan, ${ }^{4}$ Department of Obstetrics and Gynecology, Chang Gung \\ Memorial Hospital, Taoyuan, Taiwan, ${ }^{5}$ Department of Obstetrics and Gynecology, Taipei Medical University Hospital, \\ Taipei, Taiwan
}

OPEN ACCESS

Edited by:

José Mordoh

IIBBA-CONICET Leloir Institute

Foundation, Argentina

Reviewed by:

Estrella Mariel Levy,

Consejo Nacional de Investigaciones

Científicas y Técnicas

(CONICET), Argentina

Rupsa Basu,

TechnoVax Inc, United States

*Correspondence:

Jiantai Timothy Qiu

191010@h.tmu.edu.tw

Specialty section:

This article was submitted to Vaccines and Molecular Therapeutics,

a section of the journal

Frontiers in Immunology

Received: 01 March 2020

Accepted: 21 April 2020

Published: 27 May 2020

Citation:

Alson D, Schuyler SC, Yan B-X,

Samimuthu K and Qiu JT (2020)

Combination Vaccination With Tetanus

Toxoid and Enhanced Tumor-Cell

Based Vaccine Against Cervical

Cancer in a Mouse Model.

Front. Immunol. 11:927.

doi: 10.3389/fimmu.2020.00927
Cervical cancer is the fourth most common cancer in women with an estimated 570,000 new cases in 2018 which constitute about 6. 6\% of all cancers in women according to WHO report 2018. Approximately $90 \%$ of the 270,000 deaths from cervical cancer in 2015 occurred in low- and middle-income countries. In cervical cancers, which is caused by human papillomavirus (HPV) infection, the expression of HPV 16 E6 and E7 proteins are essential for tumor cell transformation and maintenance of malignancy. Prophylactic vaccines against cervical cancer caused by human papillomavirus have not proven successful. Although virus-like particle-based (VLPS) vaccines have been developed with prophylactic activities to prevent most HPV infections, the therapeutic effect of VLP vaccines has yet to be demonstrated for those who were already infected. A recent study showed that pre-conditioning mice with a potent antigen such as tetanus toxoid significantly improves lymph node homing and efficacy of dendritic cells. Tetanus toxoid has also been used in combination with DNA vaccines designed from tumor based antigens. In the present study, we pre-conditioned mice with tetanus toxoid followed by vaccination with a Granulocyte-Macrophage Colony-Stimulating Factor (GM-CSF) overexpressing tumor-cell based vaccine (GVAX). We observed that pre-conditioning with tetanus toxoid followed by vaccination with GVAX regressed tumor growth and enhanced the overall survival of the mice. Pre-conditioning with tetanus toxoid enhanced the immune response which was observed by enlarged spleen size, higher proliferation rate of lymphocytes, a higher level of IFN- $\gamma$, TNF- $\alpha$, and IL-4 antigen-specific secretions by the splenocytes. Pre-conditioning with tetanus toxoid increased memory $T$ cell migration into the tumor site and spleen. The antigen-specific cytotoxic T cell lysis percentage was also found to be higher in the group of mice vaccinated with the combination of tetanus toxoid and GVAX. Hence, pre-conditioning with tetanus toxoid prior to vaccination with a tumor-cell based vaccine overexpressing GM-CSF might be an effective strategy for targeting E7-specific HPV-associated cervical malignancy.

Keywords: human papillomavirus, vaccination, granulocyte macrophage-colony stimulating factor, cervical cancer, tetanus toxoid 


\section{INTRODUCTION}

Cervical cancer is the fourth most common malignancy in women with an estimated 260,000 deaths every year worldwide (1). Human papilloma virus (HPV) types 16 and 18 account for roughly $70 \%$ of all cervical cancer cases $(2,3)$. The HPV early proteins E6 and E7 are the main mediators for the induction of HPV-associated cervical cancer as both the proteins are consistently expressed in HPV-associated cervical cancer cells $(4,5)$. E6 inactivates the tumor suppressor p53 by binding to the ubiquitin ligase E6AP and inhibits the p53mediated signaling pathway (6-9), whereas E7 binds with pRb and promotes its proteasomal degradation (10-12). Since E6 and E7 proteins are constitutively expressed in HPV-associated cervical cancer cells, these tumor-specific antigens serve as an attractive therapeutic strategy to target HPV-associated cervical cancer cells.

Polyvalent vaccines, such as irradiated whole-cell vaccines, can activate $\mathrm{T}$ cells and cells of the innate immune system such as natural killer cells, macrophages, and eosinophils (1214). The advantage of using a whole-cell vaccine, rather than a single peptide or protein as a vaccine, is that whole-cell vaccines provide all potential antigens present in the tumor cell (15-17). Whole-cell vaccines were also developed by genetically modifying the cells that express cytokines to induce an elevated immune response to the injected irradiated tumor cells (1719). Currently available prophylactic vaccines developed from HPV virus-like particles (VLPs) against HPV-associated cervical cancer are successful in preventing tumor growth but are unsuccessful in eradicating the already infected cells $(20,21)$. Although prophylactic vaccines are successful in generating antibodies, a cellular immune response against E6 and E7 is required to eliminate already infected cells $(22,23)$. Hence, the development of new vaccine approaches is necessary to eradicate cells already infected with HPV.

Bacteria and bacterial antigens have been used in cancer immunotherapy research for many years. Coley's toxin, which consists of a mixture of inactivated Streptococcus pyogens and Serratia marcescens showed promising results by inducing tumor regression (24-26). A recent study showed that preconditioning mice and patients with bacterial antigens such as tetanus toxoid followed by vaccination with tumor antigen-specific vaccines enhanced dendritic cell migration and overall survival of both mice and humans (27). These results indicate the significance of using bacterial antigens to enhance the immune response and for promoting the regress of tumor growth.

In the present study we evaluated the immune response of mice vaccinated with tetanus toxoid and irradiated TC-1 cells, a model cell for HPV driven tumorigenesis, by engineering the TC-1 cells to secrete codon-optimized GM-CSF (GVAX). We assessed the efficacy of tetanus toxoid and tumor cellbased combination vaccination to suppress tumor growth and assessed the overall survival of the mice. Our results showed that combination vaccination with tetanus toxoid and GVAX regressed tumor growth via the Th1 and Th2 cell cytokine responses and increased the overall survival of the mice. In addition, the combination vaccination induced a higher percentage of memory $\mathrm{T}$ cells and elevated the generation of cytotoxic effector T cells in vivo.

\section{MATERIALS AND METHODS}

\section{Mice}

Female C57BL/6 (B6) mice were purchased from the National Laboratory Animal Center (Taipei, Taiwan) and housed under specific pathogen-free conditions at the animal facility of Chang Gung University. All the mice used for the experiments were of an age between 8 and 12 weeks and were kept in individually ventilated cages. All the experiments were performed in accordance with the Animal Experimental Ethics Committee of Chang Gung University. The statement of approval from the Ethics Committee contains the ethical code "CGU107-288" with the date 1st August, 2019.

\section{Cell Lines}

The TC-1 cells were engineered by the transformation of primary $\mathrm{C} 57 \mathrm{BL} / 6$ mouse lung epithelial cells with HPV type16, E6/E7 oncogenes and an activated $H$-ras oncogene, as described previously (28). Stable TC-1 cells expressing wild-type GM-CSF (wt-GM-CSF) or codon-optimized GM-CSF (cGMCSF) (GVAX) were established by lentiviral infection of TC-1 cell lines as previously described (29-31). The cell lines were maintained in RPMI 1640 medium (Gibco, Waltham, MA, USA) supplemented with $2 \mathrm{mM}$ L-glutamine, $25 \mathrm{mM}$ HEPES, $24 \mathrm{mM}$ sodium biocarbonate, $10 \%$ heat-inactivated fetal bovine serum (Invitrogen, Waltham, MA, USA), $100 \mathrm{U} / \mathrm{mL}$ penicillin, 100 $\mathrm{mg} / \mathrm{mL}$ streptomycin, and $50 \mu \mathrm{M} \beta$-mercaptoethanol at $37^{\circ} \mathrm{C}$ in an atmosphere of $5 \% \mathrm{CO}_{2}$.

\section{Tumor Model and Vaccination}

For the in vivo tumor protection experiments, C57BL/6 mice ( $n=5$ per group) were immunized intramuscularly with $1 \mathrm{Lf}$, $100 \mu l$ tetanus toxoid (Kuo Kwang, Taichung, Taiwan) into the quadriceps muscle of each mouse. Two weeks later, a booster dose was given intramuscularly. Seven days after the booster vaccination, the mice were immunized subcutaneously in the dorsal flank with $4 \times 10^{6}$ irradiated (10,000 cGy) TC-1/cGMCSF cells. Two weeks later, a booster dose of TC-1/cGM-CSF cells was given. Seven days after the final vaccination, the immunized mice were subcutaneously challenged with $2 \times 10^{5}$ TC- 1 cells in the right dorsal flank. Tumor growth was monitored three times a week using calipers and tumor volume was calculated using the formula: Length $\mathrm{x}(\text { width })^{2} \times 0.5$. When the tumor growth exceeded $2 \mathrm{~cm}$ in diameter, the mice were considered dead from the tumor burden and were subsequently euthanized (Figure A1).

\section{Spleen Weight Index and Splenocyte Proliferation}

The spleens and lymph nodes from immunized mice were aseptically harvested, transferred to six-well culture plate containing RPMI 1640 medium supplemented with 10\% fetal bovine serum and penicillin $(10 \mathrm{U} / \mathrm{mL})$ and weighted. Spleen indices were calculated as organ weight (milligram, mg) per 
gram of mouse body weight. The splenocytes $\left(5 \times 10^{5}\right.$ cells/well $)$ were incubated with $10 \mu \mathrm{g} / \mathrm{mL}$ of E7 peptide or medium alone (unstimulated) in triplicate for $72 \mathrm{~h}$ at $37^{\circ} \mathrm{C}$. The proliferation activity was examined by a CFSE cell proliferation assay kit (Thermo Fisher Scientific, Waltham, MA, USA).

\section{Cytokine Secretion Measurement With ELISA}

GM-CSF, IFN- $\gamma$, TNF- $\alpha$, and IL-4 levels from the secreting splenocytes were measured by ELISA with a commercially available ELISA kit (Biolegend, San Diego, CA, USA). A total of $1 \times 10^{6}$ TC- 1 cells were cultured in $7 \mathrm{~mL}$ of the medium for $24 \mathrm{~h}$ in a $10 \mathrm{~cm}$ dish for GM-CSF analyses. Supernatants were collected by centrifugation at 220x rcf for $4 \mathrm{~min}$ and diluted appropriately for the assay. To analyze IFN- $\gamma$, TNF- $\alpha$, and IL4 , splenocytes were harvested from the mice, stimulated with E7 peptide for $72 \mathrm{~h}$. Standards or experimental samples were added to microtiter plates coated with monoclonal antibody to the cytokine of interest and incubated for $2 \mathrm{~h}$ with shaking. After washing, horseradish peroxidase-conjugated, cytokine specific antibody was added to each well and incubated for $1 \mathrm{~h}$ and washed. Substrate solution was added and incubated for $30 \mathrm{~min}$ with shaking and the reaction was stopped using stop solution. Cytokine levels were determined by measuring the optical density at $450 \mathrm{~nm}$ by using microtiter plate reader (Molecular Devices, San Jose, CA, USA).

\section{Flow Cytometric Analyses of Immune Cells}

For memory $\mathrm{T}$ cell analyses, splenocytes from various vaccinated groups were harvested, lysed with $1 \mathrm{x}$ RBC lysis buffer, washed and $1 \times 10^{6}$ cells per well was utilized for each reaction followed by flow cytometry (BD FACSCaliber, Hampton, NH, USA) analysis. Splenocytes were stained with anti-CD44-APC (Biolegend, San Diego, CA, USA), anti-CD4-FITC (Biolegend, San Diego, CA, USA), anti-CD8-PE (Biolegend, San Diego, CA, USA), anti-CD25-APC (Biolegend, San Diego, CA, USA), or anti-Foxp3-APC (Biolegend, San Diego, CA, USA).

\section{In vivo Cytotoxicity Assays}

A total of $10^{7}$ spleen cells from naïve syngeneic spleen cells from wild type C57BL/6 mice used as in vivo target cells. Cells were labeled with either $5 \mathrm{M}$ carboxyflourescein succinimidyl ester $\left(\mathrm{CFSE}^{\text {high }}\right.$ ) or $0.5 \mathrm{M}$ CFSE $\left(\mathrm{CFSE}^{\text {low }}\right.$ ) for $15 \mathrm{~min}$ at $37^{\circ} \mathrm{C}$ and washed twice with phosphate-buffered saline (PBS). CFSE ${ }^{\text {high }}$ cells were incubated with MHC Class-I restricted E7 peptide $(10 \mu \mathrm{g} / \mathrm{mL})$ or MHC Class-II restricted E7 peptide $(10 \mu \mathrm{g} / \mathrm{mL})$ in CTL medium for $1 \mathrm{~h}$ at $37^{\circ} \mathrm{C}$. CFSE ${ }^{\text {low }}$ cells were incubated with CTL medium without peptide, serving as an internal control. CFSE labeled cells were then washed twice with PBS. A mixture of $2.5 \times 10^{7} \mathrm{CFSE}^{\text {high }}$ and CFSE ${ }^{\text {low }}$ cells were injected intravenously through the tail vein. After $12 \mathrm{~h}$ of in vivo incubation, splenocytes were harvested and single cell suspensions were analyzed for detection and quantification of CFSE-labeled cells. The percent specific lysis was determined by the ratio of recovered nonpeptide-treated control spleen cells to peptide-sensitized spleen cells (percentage of CFSE ${ }^{\text {low }}$ cells/percentage of CFSE $^{\text {high }}$ cells).

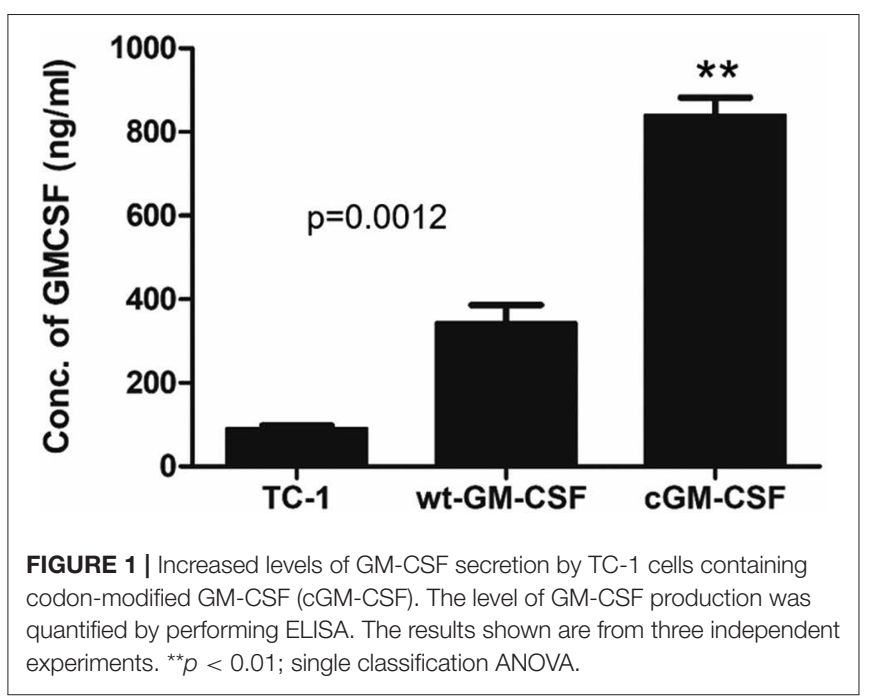

\section{Statistical Analyses}

All the analyses were performed using GraphPad Prism statistical software (Graph Pad Software, La Jolla, CA, USA). One-way ANOVA and log-rank (Mantel-Cox) tests were used to analyze the tumor growth and mouse survival data, respectively. A value of ${ }^{*} p<0.05,{ }^{* *} p<0.01,{ }^{* * *} p<0.001$ were considered statistically significant.

\section{RESULTS}

\section{Higher Levels of GM-CSF Secretion by TC-1/cGM-CSF Stable Cell Lines}

To check whether stable cell lines can enhance the level of GM-CSF compared with other cell lines such as TC-1 cells and TC-1 cell expressing wild-type GM-CSF (TC-1/wt-GM-CSF) we measured the levels of protein using TC- 1 cell expressing codon-optimized GM-CSF (TC-1/cGM-CSF) by ELISA. Cells were grown in culture media, and medium containing GM-CSF was collected from the cells cultured for $24 \mathrm{~h}$ to perform ELISA. As shown in Figure 1, TC-1 cells infected with lentivirus cGMCSF produced increased levels of GM-CSF compared with the TC-1 cells infected with lentivirus wt-GM-CSF. These results show that GM-CSF is expressed more effectively when its codons are optimized.

\section{Vaccination With Tetanus Toxoid and GVAX Induced Enhanced Splenocyte Proliferation} Seven days after the final vaccination, spleens were isolated and weighed (Figure A1). As shown in the Figures 2A,B mice vaccinated with combination of tetanus toxoid and TC-1/cGMCSF (GVAX) had much larger spleen weight indices compared with the mice vaccinated with either tetanus toxoid or GVAX alone. The spleen weight index is presented as the ratio of spleen weight to the mouse body weight. This indicates that combination vaccination with tetanus toxoid and GVAX might induce higher levels of splenocytes proliferation. We then evaluated the E749-57 peptide specific proliferation under 

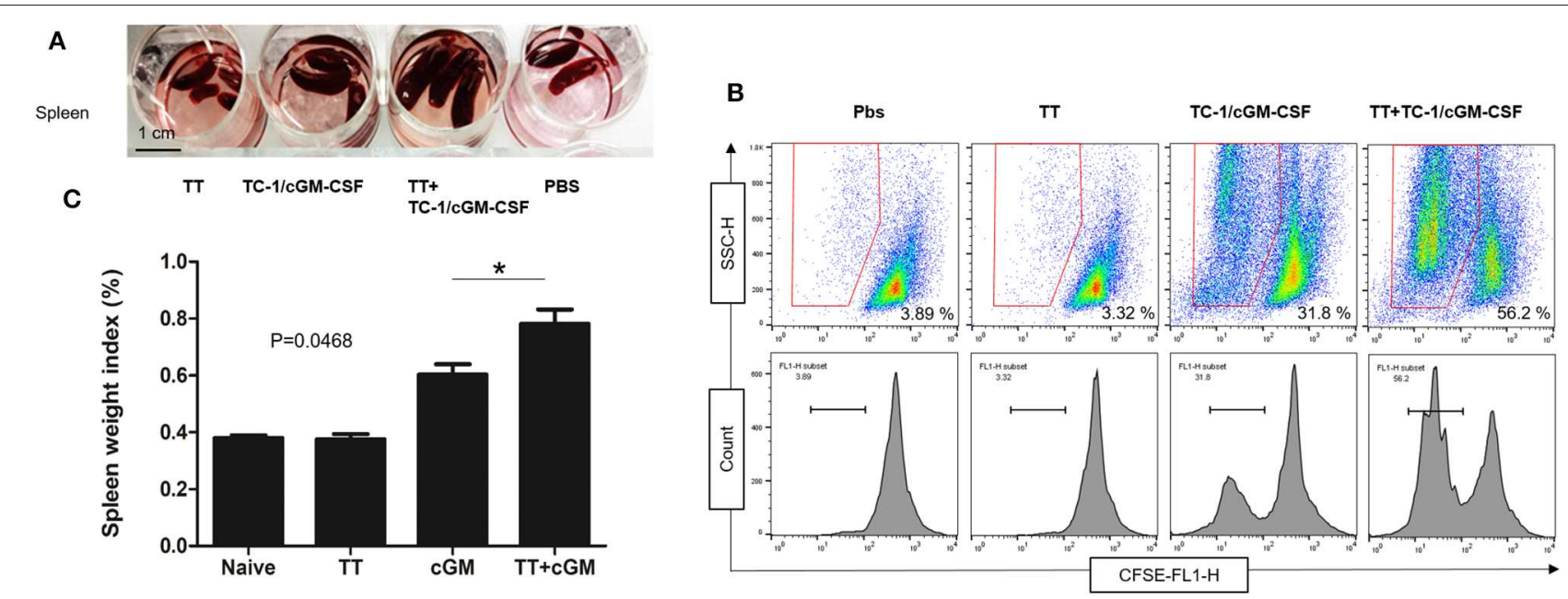

FIGURE 2 | Enhanced splenocyte proliferation after combination vaccination. C57BL/6 mice $(n=5)$ were injected with tetanus toxoid or irradiated TC-1/cGM-CSF cells (GVAX) or combination of both $\left(4 \times 10^{6}\right)$ by intramuscular and subcutaneously injections at the dorsal flank. Seven days after the last immunization, the spleens from the mice were harvested weighed and analyzed for splenocyte proliferation. (A) The spleens from immunized mice were isolated and weighed. (B) Spleen weight index was calculated as organ weight per gram of mouse body weight. (C) The proliferation activity of splenic lymphocytes was measured under stimulation with E7 peptide. ${ }^{*} p<0.05$; single classification ANOVA.

stimulation with the E749-57 peptide employing a CFSE cell proliferation assay, and found that the splenocytes from the group of mice that had received the combination vaccination of tetanus toxoid and GVAX (56.2\%) showed significantly higher proliferative activity than that from the GVAX alone (31.8\%) group or tetanus toxoid alone group (3.32\%). The lymphocytes from PBS group showed no obvious proliferation activity against the E749-57 peptide. This result shows that the spleen weight index was positively correlated with the splenocyte proliferation percentage.

\section{Increased Levels of Th1 Cell and Th2 Cell Cytokines in Mice Vaccinated With the Combination Vaccination}

Th1 cell and Th2 cell immunity is critically important for the induction of anti-tumor cellular immunity $(32,33)$. Th1 cells secrete cytokines such as IFN- $\gamma$, TNF- $\alpha / \beta$, IL-2, and IL-10 (34). Th2 cells secrete different types of cytokines such as IL-10, IL4, IL-5, and IL-6 $(35,36)$. To determine whether combination vaccination influences Th1 and Th2 cytokine production, we have analyzed the levels of IFN- $\gamma$, TNF- $\alpha$, and IL- 4 responses in the culture supernatants of isolated cells from spleens which were stimulated with HPV 16 E749-57 peptide for $72 \mathrm{~h}$. ELISA was performed to analyze the medium collected from cultured splenocytes (Figure A1). As shown in the Figures 3A-C, there was a significant increase in the levels of IFN- $\gamma$ (mean $=670$ $\mathrm{pg} / \mathrm{mL}) \mathrm{TNF}-\alpha($ mean $=592 \mathrm{pg} / \mathrm{mL})$, and IL-4 levels $($ mean $=79$ $\mathrm{pg} / \mathrm{mL}$ ) secreted by the splenocytes after $72 \mathrm{~h}$ of peptide induction in the group of mice vaccinated with combination vaccination of tetanus toxoid and GVAX compared with the group of mice vaccinated with either tetanus toxoid (IFN- $\gamma$ mean $=95.32$ $\mathrm{pg} / \mathrm{mL}, \mathrm{TNF}-\alpha$ mean $=0.39 \mathrm{pg} / \mathrm{mL}, \mathrm{IL}-4$ mean $=5 \mathrm{pg} / \mathrm{mL}$ ) or GVAX $($ IFN $-\gamma$ mean $=381.59 \mathrm{pg} / \mathrm{mL}$, TNF- $\alpha$ mean $=209 \mathrm{pg} / \mathrm{mL}$,
IL-4 mean $=38 \mathrm{pg} / \mathrm{mL}$ ) alone. There was no significant difference in the level of secretion of cytokines such as IFN- $\gamma$, TNF- $\alpha$ and IL- 4 in the group of mice either vaccinated with PBS or with tetanus toxoid.

\section{Higher Percentage of Memory T Cell Generation in the Spleen After Combination Vaccination of Tetanus and GVAX}

Activation of both CD4+ and CD8+ T lymphocytes is important for an efficient immune response to destroy tumor cells (37, 38). CD44 expression is upregulated in naïve $\mathrm{T}$ cells after their activation. Memory $\mathrm{T}$ cells have intermediate to high expression of CD44. We initially analyzed the mechanism of action of the combination vaccination by measuring the generation of CD44+ memory $\mathrm{T}$ cells in the tumor-infiltrating lymphocyte (TIL) population. Thirty days after the inoculation with live TC-1 tumor cells subcutaneously, TILs were harvested from the dissected tumor samples (Figure A1). As shown in Figures 4A,B we detected a higher percentage of memory $\mathrm{T}$ cells double positive for CD8 and CD44 T surface markers in the group of mice vaccinated with both tetanus toxoid and GVAX vaccination $(12.5 \%)$ compared with the group of mice vaccinated with either tetanus toxoid $(2.47 \%)$ or GVAX $(8.24 \%)$ alone. Similarly, we also detected a higher percentage of CD4+ and CD44+ double positive memory $\mathrm{T}$ cell population $(5.67 \%)$ in the group of mice vaccinated with the combination vaccination of tetanus toxoid and GVAX (Figure 4A). We also analyzed the spleens and detected a higher percentage of CD8+ and CD44+ double positive memory $\mathrm{T}$ cell population $(5.67 \%)$ in the group of mice vaccinated with the combination vaccination of tetanus toxoid and GVAX (Figure 4B). 

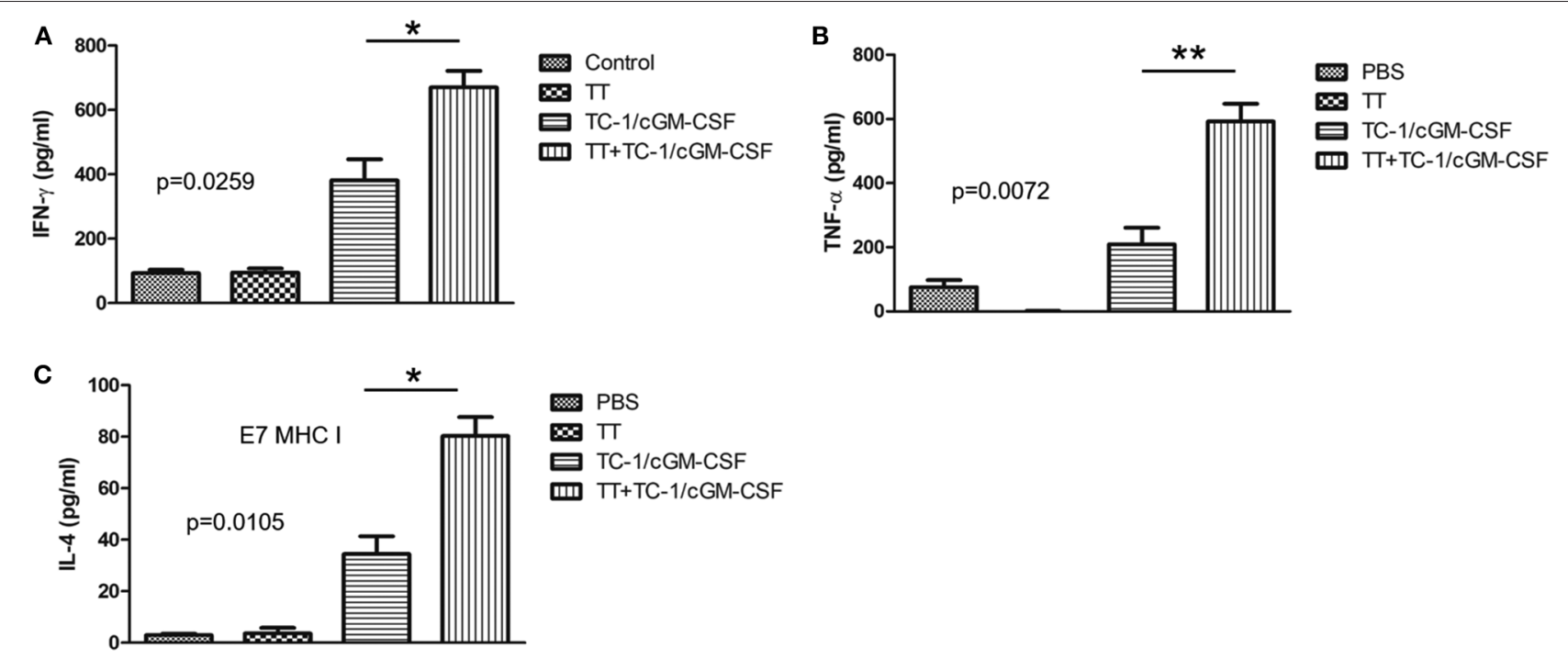

FIGURE 3 | Combination of tetanus toxoid and GVAX vaccination enhances the level of Th1 cell and Th2 cell cytokine secretions. C57BL/6 mice ( $n=5$ ) were injected with tetanus toxoid or irradiated TC-1/cGM-CSF cells or combination of both $\left(4 \times 10^{6}\right)$ by intramuscular and subcutaneously injections at the dorsal flank. Seven days after the last immunization, the spleens from the mice were harvested, cultured with E7 peptide and analyzed for Th1 and Th2 cytokine secretion. The results depict the stimulated secretion of (A) IFN- $\gamma$, (B) TNF- $\alpha$, and (C) IL-4, by splenocytes. The data are presented as the mean $\pm \mathrm{SD}$ of triplicates. ${ }^{*} p<0.05$; ${ }^{* *} p<0.01$; single-classification ANOVA.

\section{Generation of Cytotoxic Effector T Cells in vivo After Combination Vaccination With Tetanus Toxoid and GVAX}

We next evaluated the ability of tetanus toxoid and GVAX combination vaccine to generate $\mathrm{E}_{49-57}$ specific cell CTL response in an in vivo assay. Anti-tumor function of CD8+ $\mathrm{T}$ cells depends on the generation of effector $\mathrm{T}$ cells. Seven days after the final vaccination, mice were injected intravenously with a combination of peptide-pulsed (CFSE ${ }^{\text {high }}$ ) and peptideunpulsed $\left(\mathrm{CFSE}^{\text {low }}\right)$ splenocytes and the spleens were harvested after $14 \mathrm{~h}$ of in vivo incubation. As shown in Figure 5, E7 $49-57$ specific lysis was detected in TC-1/cGM-CSF vaccinated animals but mice vaccinated with TC- $1 / \mathrm{cGM}$-CSF and tetanus toxoid combination vaccination generated higher specific lytic activities $(\mathrm{R}=2.34,57.36 \%)$ compared with mice vaccinated with tetanus toxoid $(\mathrm{R}=1.15,13 \%)$ or $\mathrm{TC}-1 / \mathrm{cGM}-\mathrm{CSF}(\mathrm{R}=1.81,44.8 \%)$ vaccine alone. The ratio between the percentages of un-pulsed vs $\mathrm{E}_{49-57}$ peptide-pulsed (CFSE ${ }^{\text {low }} / \mathrm{CFSE}^{\text {high }}$ ) was calculated to obtain the numerical valve for cytotoxicity. These data indicate that $\mathrm{E}_{49-57}$ peptide can stimulate CD8 + T cell differentiation into CTL effector cells in vivo.

\section{Combination Vaccination With Tetanus Toxoid and GVAX Induced Enhanced Immuno-Surveillance and Effectively Inhibits Tumorigenesis in vivo}

To evaluate the anti-tumor efficacy of combination vaccination with tetanus toxoid and GVAX on tumor growth, mice were pre-conditioned twice with tetanus toxoid at two-week intervals followed by vaccination with GVAX at two-week time intervals.
The mice were challenged with $2 \times 10^{5}$ TC- 1 tumor cells 7 days after the final vaccination (Figure A1). The tumor volume was measured weekly thrice. We observed an inhibition of subcutaneous tumor growth 10 days after the tumor challenge in the group of mice vaccinated with combination vaccination (Figures 6A-C). Group of mice vaccinated with either tetanus toxoid or GVAX alone revealed rapid subcutaneous tumor growth. We observed that group of mice vaccinated with combination vaccination showed TC-1 tumor regression in $90 \%$ of the mice, and these mice remained tumor free over the test period. We also observed an increase in the life span for over 53 days in the mice vaccinated with combination vaccination of tetanus toxoid and GVAX compared with those of the group of mice vaccinated with either tetanus toxoid or TC-1/cGMCSF tumor cell-based vaccine (Figure 6D). These results showed that mice vaccinated with the combination of tetanus toxoid and GVAX displayed the most efficient TC-1 tumor regression compared with mice that had been vaccinated with either tetanus toxoid or GVAX alone.

\section{DISCUSSION}

This study shows that in a cervical cancer mouse model vaccination with a combination vaccine such as tetanus toxoid and TC-1/cGM-CSF (GVAX) regressed tumor growth and enhanced the overall survival of mice compared with mice receiving either tetanus toxoid or GVAX alone. We also found that combination vaccination of tetanus toxoid and GVAX can enhance Th1 and Th2 cell cytokine response and generate memory $T$ cells. Finally, we showed that combination vaccination with tetanus toxoid and GVAX can generate higher percentage of 

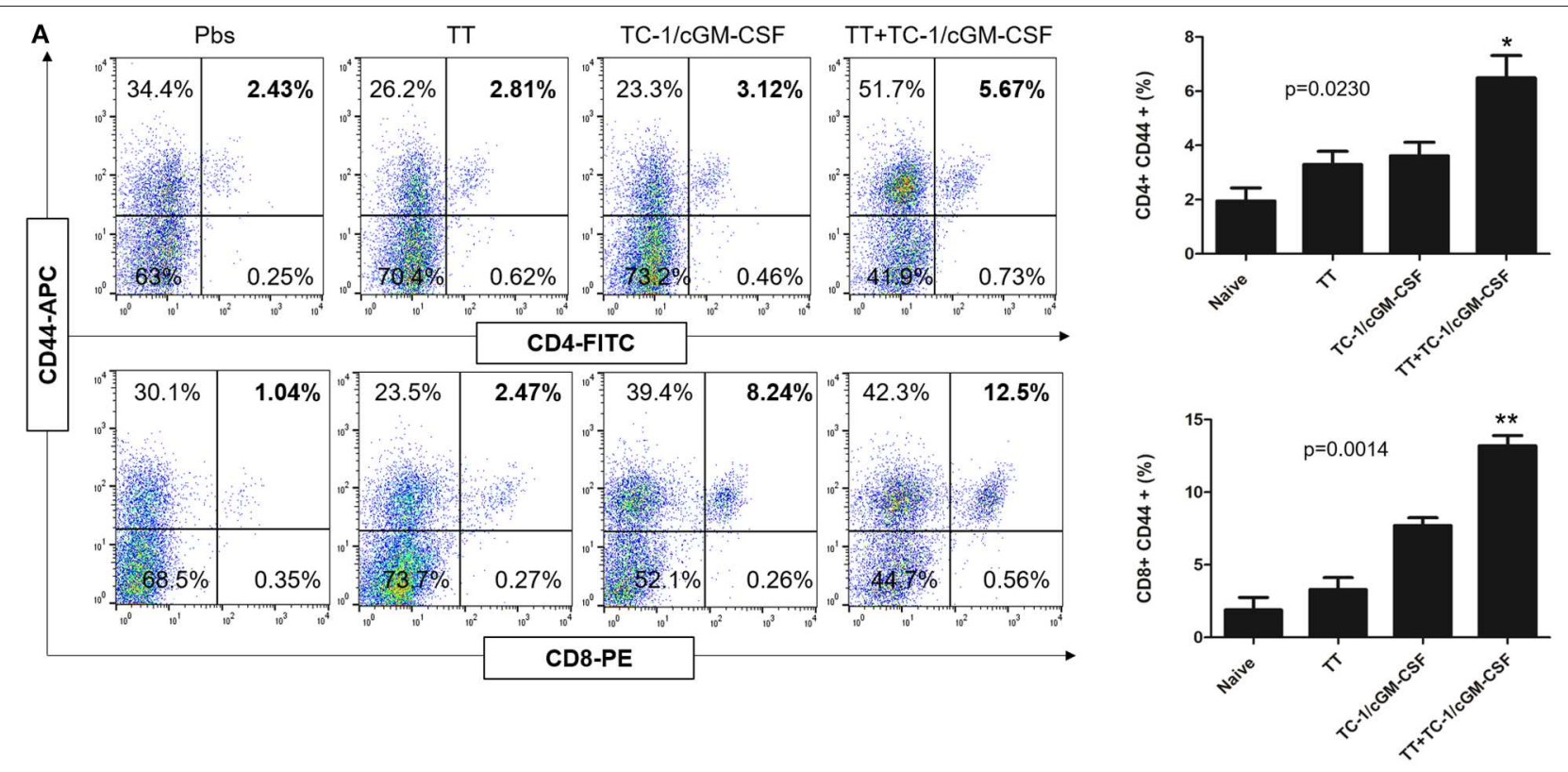

B
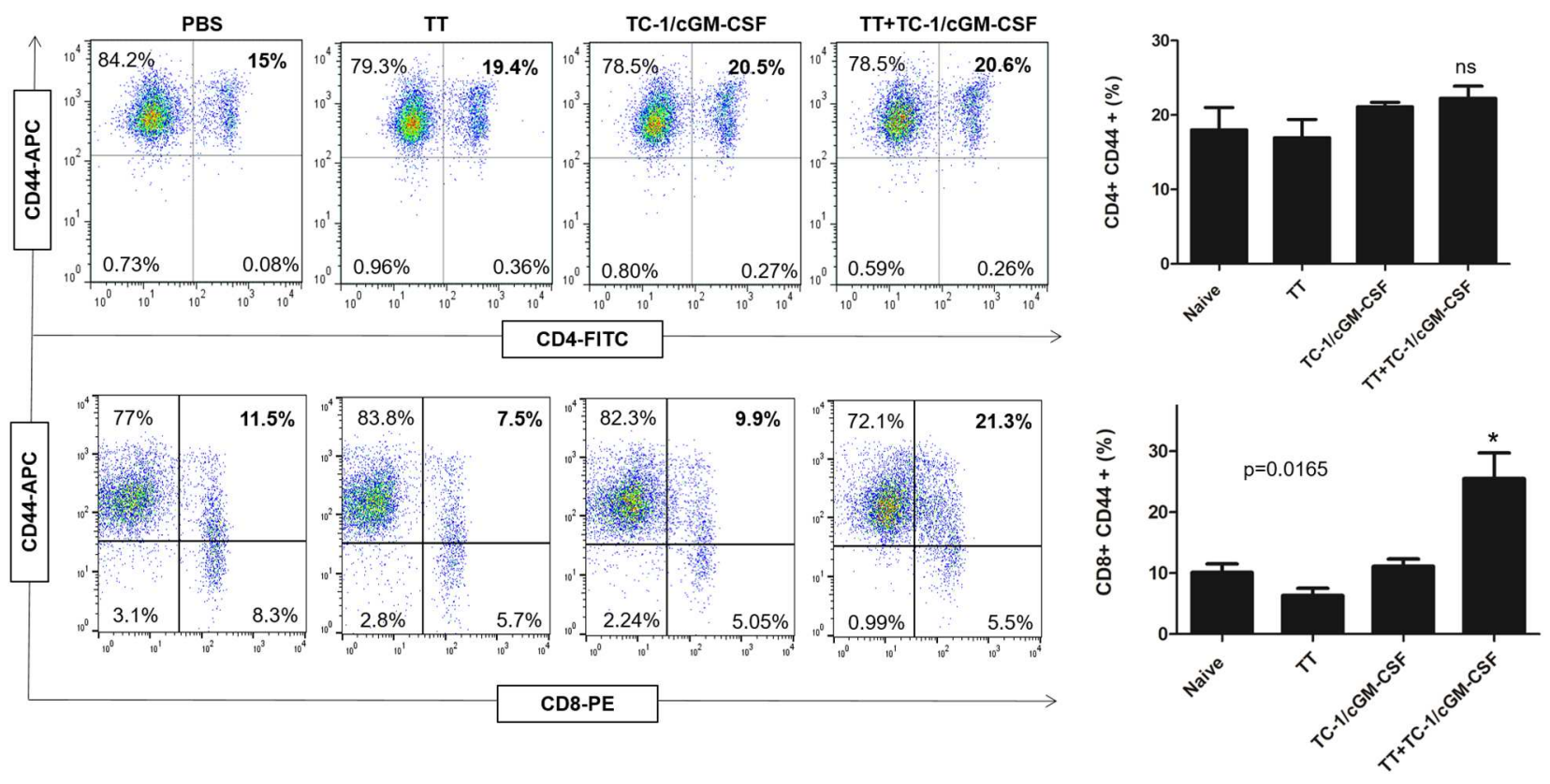

FIGURE 4 | Combination vaccination increased the percentage of CD8+ CD44+ memory T cell lymphocyte in TILs and spleen. C57BL/6 mice $(n=5)$ were injected with tetanus toxoid or GVAX or combination of both $\left(4 \times 10^{6}\right)$ by intramuscular and subcutaneously injections at the dorsal flank. Seven days after the last immunization, the mice were inoculated with $2 \times 10^{5} \mathrm{TC}-1$ cells subcutaneously at the right flank. 30 days after the tumor inoculation, the tumor tissues and spleens were harvested for analysis. (A) Representative flow cytometry plots of percentages of CD4+ CD44+ and CD8+ CD44+ memory T cells in the TILs. The bar graph shows the percentage of indicated population of total CD4+ CD8+ CD44+ memory T cells. (B) Representative flow cytometry plots of percentages of CD4+ CD44+ and CD8+ CD44+ memory T cells in the spleen. The bar graph shows the percentage of indicated population of total CD4+ CD8+ CD44+ memory T cells. The data are presented as the mean $\pm \mathrm{SD}$ of duplicate values. ${ }^{*} p<0.05$; ${ }^{* *} p<0.01$; single-classification ANOVA.

cytotoxic effector T cells in vivo compared with mice vaccinated with tetanus toxoid or GVAX alone.

Attenuated bacterial antigens play an important role in immunotherapy as they regulate various immune cells such as tumor-associated macrophages, dendritic cells, tumorassociated lymphoid cells such as NK cells, CD4+, and CD8+ cells and regulatory $\mathrm{T}$ cells $(39,40)$. A recent study in a Glioblastoma tumor model showed that pre-conditioning 

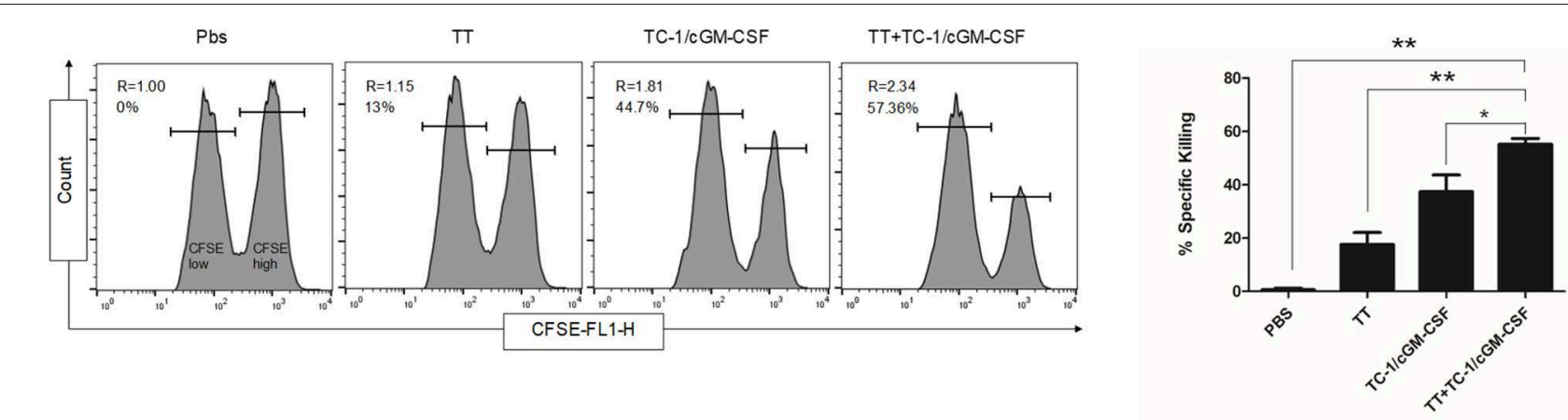

FIGURE 5 | Effects of combination vaccination on the generation E7-specific CTL in C56BL/6 mice. Mice were administered intramuscularly with 1Lf of the tetanus toxoid with a two-week interval of time followed by subcutaneous injection with GVAX twice with one-week time interval. To analyze E7-specific cytotoxicity, cells pooled from the spleen of naive mice were pulsed with E7 [aa-49-57, RAHYNIVTF] peptide and labeled with a high concentration of CFSE (CFSE high) and low concentration of CFSE (CFSE ${ }^{\text {low }}$ ). A 1:1 mixture of each target cell population was injected into recipient mice and specific cytotoxicity was determined $14 \mathrm{~h}$ later. Representative histograms of the spleen cells of the mice in each group are shown with percentages of the specific killing of E7 [aa-49-57, RAHYNIVTF] peptide-pulsed target cells in the spleens. The experiments were repeated two times with five animals per group. The data are presented as mean \pm SD of duplicate values. ${ }^{*} p<0.05 ;{ }^{* *} p<0.01$; single-classification ANOVA.
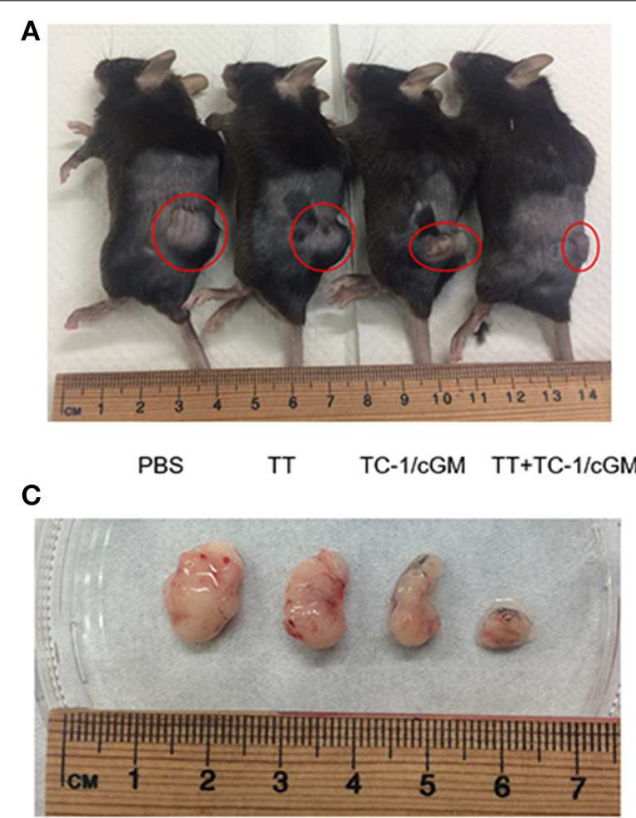

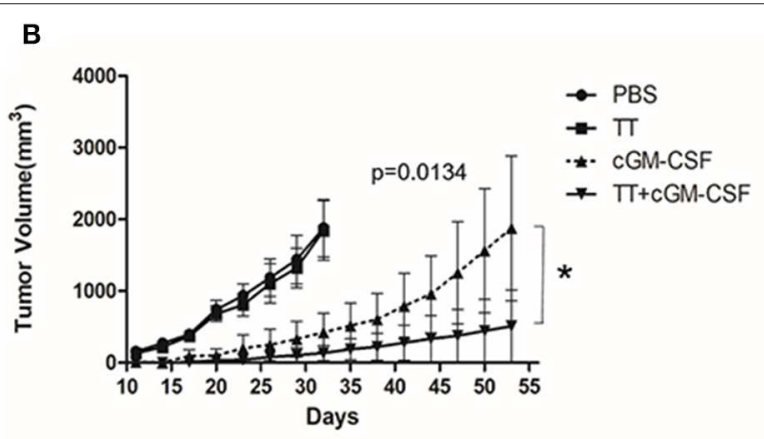

D

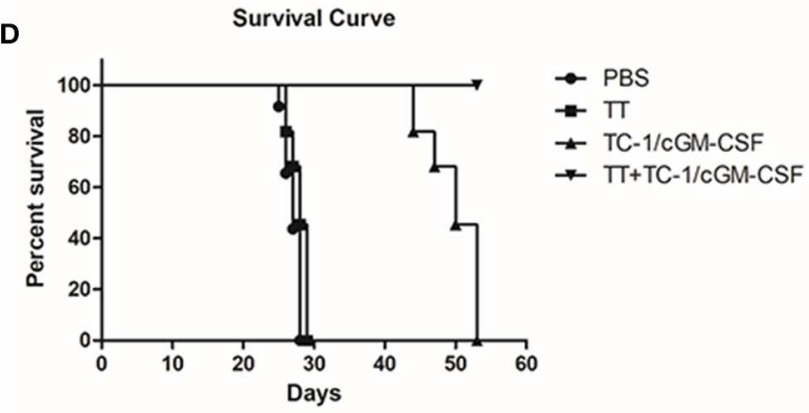

FIGURE 6 | Combination vaccination with tetanus toxoid and GVAX can effectively inhibit tumor growth. (A) C57BL/6 mice $(n=5)$ were injected with tetanus toxoid or GVAX or combination of both $\left(4 \times 10^{6}\right)$ subcutaneously at the dorsal flank. Seven days after the last immunization, the mice were inoculated with $2 \times 10^{5}$ TC- 1 cells subcutaneously at the right flank. Representative mice bearing xenograft TC-1 tumor. (B) Comparison of tumors excised from xenograft mice (C). The tumor size was monitored weekly twice for 7 weeks. The line graph depicts tumor volumes over time in various vaccinated mouse groups. (D) Survival curve with mice $(n=5)$ vaccinated with PBS, tetanus toxoid, GVAX and combination of tetanus toxoid and GVAX. The data are presented as the mean \pm SD of triplicate values. The log rank (Mantel-Cox) test was used to compare the survival rates among various groups. ${ }^{*} p<0.05$; single-classification ANOVA.

mice with tetanus toxoid followed by dendritic cell-based vaccination pulsed with Cytomegalovirus phosphoprotein 65 (pp65) can enhance dendritic cell migration bilaterally and significantly improved overall survival (27). These studies showed the significance of bacterial antigens in the field of cancer immunotherapy.
Pro-inflammatory cytokines such as IFN- $\gamma$ and TNF- $\alpha$ can help stimulate effector cells and enhance tumor cell recognition of cytotoxic effector cells $(41,42)$. Consistent with this knowledge, our study shows that combination vaccination with tetanus toxoid and GVAX can induce higher level of IFN- $\gamma$ and TNF- $\alpha$ compared with the group of 
mice vaccinated with either tetanus toxoid or TC-1/cGMCSF vaccine alone. We also observed a higher percentage of cytotoxic effector cells in the group of mice vaccinated with the combination vaccination compared with that of the control in vivo.

Surprisingly we found a variation in the size of spleens in mice vaccinated with different vaccines and among them mice vaccinated with a combination of tetanus toxoid and GVAX exhibit a higher spleen size compared with the group of mice vaccinated with either tetanus toxoid or GVAX alone. Studies have shown that bigger spleen sizes might be due to higher proliferation rates of the splenocytes after vaccination. Lymphocytes proliferate when it comes in contact with antigenpresenting cells containing peptide-MHC complexes. Generally, splenic populations trap and remove blood antigens and initiate innate and adaptive immune responses against pathogens.

In summary, we demonstrated that combination vaccination with tetanus toxoid and GVAX can elicit a greater anti-tumor immune response in mice compared with those of mice receiving the tetanus toxoid or TC-1/cGM-CSF vaccine alone. In addition, combination vaccination induced long-lasting memory $\mathrm{T}$ cell responses of cytolytic effector $\mathrm{T}$ cells compared with the control groups. Hence, pre-conditioning with tetanus toxoid prior to vaccination with a tumor-cell based vaccine overexpressing GMCSF might be an effective strategy to target E7-specific HPVassociated cervical malignancy.

In conclusion, bacterial based antigens such as tetanus toxoid in combination with tumor antigen can be used effectively to enhance the anti-tumor effects and immunestimulatory capacity of the host infected with HPV. Although using a bacterial-based antigen for immunotherapy has few drawbacks, the promising results of this therapy must be studied extensively to prove its importance in the field of cancer immunotherapy. In this way, the options available for the cancer patients preferring for immunotherapeutic treatment widely opens up. If proven with strong results in the pre-clinical and clinical studies, HPV infected individuals can opt for bacterial immunotherapy in situations where

\section{REFERENCES}

1. Torre LA, Islami F, Siegel RL, Ward EM, Jemal A. Global cancer in women: burden and trends. Cancer Epidemiol Biomarkers Prev. (2017) 26:44457. doi: 10.1158/EPI-16-0858

2. Munoz N, Bosch FX, Castellsague X, Diaz M, de Sanjose S, Hammouda $\mathrm{D}$, et al. Against which human papillomavirus types shall we vaccinate and screen? The international perspective. Int J Cancer. (2004) 111:27885. doi: 10.1002/ijc. 20244

3. Smith JS, Lindsay L, Hoots B, Keys J, Franceschi S, Winer R, et al. Human papillomavirus type distribution in invasive cervical cancer and high-grade cervical lesions: a meta-analysis update. Int J Cancer. (2007) 121:62132. doi: $10.1002 /$ ijc. 22527

4. Schwarz E, Freese UK, Gissmann L, Mayer W, Roggenbuck B, Stremlau A, et al. Structure and transcription of human papillomavirus sequences in cervical carcinoma cells. Nature. (1985) 314:111-4. doi: 10.1038/314111a0

5. Yim EK, Park JS. The role of HPV E6 and E7 oncoproteins in HPV-associated cervical carcinogenesis. Cancer Res Treat. (2005) 37:31924. doi: $10.4143 / \mathrm{crt} .2005 .37 .6 .319$ treatment with other immunotherapeutic technique is a problem.

\section{DATA AVAILABILITY STATEMENT}

All datasets generated for this study are included in the article/Supplementary Material.

\section{ETHICS STATEMENT}

The animal studies were reviewed and approved by the Review Board of Ethics Committee of Chang Gung University. The statement of approval from the Ethics Committee contains the ethical code CGU107-288 with the date 1st August, 2019.

\section{AUTHOR CONTRIBUTIONS}

JQ and DA designed experiments, analyzed data, and discussed the data. DA, B-XY, and KS performed the experiments. DA and SS wrote the manuscript and revised the manuscript.

\section{FUNDING}

This work was supported by Chang Gung Memorial Hospital (CGMH), Linkou, under the contract numbers MOST1082314-B182-032, MOST106-2314-B182-058, CMRPD1K0121, CMRPD1J0121, BMRPC59, and CMRPD1H0601. The funders had no role in study design, data collection, and analysis, the decision to publish, or the preparation of the manuscript. Funds for open access publication with the help of Chang Gung University.

\section{SUPPLEMENTARY MATERIAL}

The Supplementary Material for this article can be found online at: https://www.frontiersin.org/articles/10.3389/fimmu. 2020.00927/full\#supplementary-material

6. Scheffner M, Huibregtse JM, Vierstra RD, Howley PM. The HPV-16 E6 and E6-AP complex functions as a ubiquitin-protein ligase in the ubiquitination of p53. Cell. (1993) 75:495-505. doi: 10.1016/0092-8674(93)90384-3

7. Scheffner M, Werness BA, Huibregtse JM, Levine AJ, Howley PM. The E6 oncoprotein encoded by human papillomavirus types 16 and 18 promotes the degradation of p53. Cell. (1990) 63:1129-36. doi: 10.1016/0092-8674(90)90409-8

8. Werness BA, Levine AJ, Howley PM. Association of human papillomavirus types 16 and 18 E6 proteins with p53. Science. (1990) 248:76-9. doi: 10.1126/science. 2157286

9. Huibregtse JM, Scheffner M. Mechanisms of tumor suppressor protein inactivation by the human papillomavirus E6 and E7 oncoproteins. Sem Vir. (1994) 5:357-67. doi: 10.1006/smvy.1994.1040

10. Boyer SN, Wazer DE, Band V. E7 protein of human papilloma virus16 induces degradation of retinoblastoma protein through the ubiquitinproteasome pathway. Cancer Res. (1996) 56:4620. doi: 10.1007/sr.32583

11. Jones DL, Thompson DA, Munger K. Destabilization of the RB tumor suppressor protein and stabilization of p53 contribute to HPV type 16 E7induced apoptosis. Virology. (1997) 239:97-107. doi: 10.1006/viro.1997.8851 
12. Oh KJ, Kalinina A, Bagchi S. Destabilization of Rb by human papillomavirus E7 is cell cycle dependent: E2-25K is involved in the proteolysis. Virology. (2010) 396:118-24. doi: 10.1016/j.virol.2009.10.018

13. Han Q, Wang Y, Pang M, Zhang J. STAT3-blocked whole-cell hepatoma vaccine induces cellular and humoral immune response against HCC. J Exp Clin Cancer Res. (2017) 36:156. doi: 10.1186/s13046-0170623-0

14. Dranoff G. GM-CSF-based cancer vaccines. Immunol Rev. (2002) 188:147-54. doi: 10.1034/j.1600-065x.2002.18813.x

15. Keenan BP, Jaffee EM. Whole cell vaccines-past progress and future strategies. Semin Oncol. (2012) 39:276-86. doi: 10.1053/j.seminoncol.2012. 02.007

16. Chiang CL, Coukos G, Kandalaft LE. Whole tumor antigen vaccines: where are we? Vaccines. (2015) 3:344-72. doi: 10.3390/vaccines 3020344

17. Srivatsan S, Patel JM, Bozeman EN, Imasuen IE, He S, Daniels D, et al. Allogeneic tumor cell vaccines: the promise and limitations in clinical trials. Hum Vaccin Immunother. (2014) 10:52-63. doi: 10.4161/hv.26568

18. Miguel A, Herrero MJ, Sendra L, Botella R, Diaz A, Algas R, et al. Antitumor cell-complex vaccines employing genetically modified tumor cells and fibroblasts. Toxins (Basel). (2014) 6:636-49. doi: 10.3390/toxins6020636

19. Maeng HM, Berzofsky JA. Strategies for developing and optimizing cancer vaccines. F1000Res. (2019) 8. doi: 10.12688/f1000research.18693.1

20. Baden LR, Curfman GD, Morrissey S, Drazen JM. Human papillomavirus vaccine - opportunity and challenge. N Engl J Med. (2007) 356:199091. doi: 10.1056/NEJMe078088

21. Yadav R, Zhai L, Tumban E. Virus-like particle-based 12 vaccines against HPVS: where are we today? Viruses. (2019) 12:18. doi: 10.3390/v12010018

22. Duggan-Keen MF, Brown MD, Stacey SN, Stern PL. Papillomavirus vaccines. Front Biosci. (1998) 3:D1192-208. doi: 10.2741/a356

23. Cornelison TL. Human papillomavirus genotype 16 vaccines for cervical cancer prophylaxis and treatment. Curr Opin Oncol. (2000) 12:46673. doi: 10.1097/00001622-200009000-00014

24. Kienle GS. Fever in cancer treatment: coley's therapy and epidemiologic observations. Glob Adv in Health Med. (2012) 1:92-100. doi: 10.7453/gahmj.2012.1.1.016

25. McCarthy EF. The toxins of William B. Coley and the treatment of bone and soft-tissue sarcomas. Iowa Orthop. (2006) 26:154-8.

26. Wiemann B, Starnes CO. Coley's toxins, tumor necrosis factor and cancer research: a historical perspective. Pharmacol Ther. (1994) 64:52964. doi: 10.1016/0163-7258(94)90023-x

27. Mitchell DA, Batich KA, Gunn MD, Huang MN, Sanchez-Perez L, Nair SK, et al. Tetanus toxoid and CCL3 improve dendritic cell vaccines in mice and glioblastoma patients. Nature. (2015) 519:366-9. doi: 10.1038/nature14320

28. Lin KY, Guarnieri FG, Staveley-O'Carroll KF, Levitsky HI, August JT, Pardoll DM, et al. Treatment of established tumors with a novel vaccine that enhances major histocompatibility class II presentation of tumor antigen. Cancer Res. (1996) 56:21-6.

29. Chang LJ, Urlacher V, Iwakuma T, Cui Y, Zucali J. Efficacy and safety analyses of a recombinant human immunodeficiency virus type 1 derived vector system. Gene Ther. (1999) 6:715-28. doi: 10.1038/sj.gt.3300895
30. Chang LJ, Zaiss AK. Self-inactivating lentiviral vectors and a sensitive Cre-loxP reporter system. Methods Mol Med. (2003) 76:367-82. doi: 10.1385/1-59259-304-6:367

31. Zaiss AK, Son S, Chang LJ. RNA 3' readthrough of oncoretrovirus and lentivirus: implications for vector safety and efficacy. J Virol. (2002) 76:720919. doi: 10.1128/jvi.76.14.7209-7219.2002

32. Nishimura T, Iwakabe K, Sekimoto M, Ohmi Y, Yahata T, Nakui M, et al. Distinct role of antigen-specific T helper type 1 (Th1) and Th2 cells in tumor eradication in vivo. J Exp Med. (1999) 190:617-28. doi: 10.1084/jem.190.5.617

33. Zhao X, Liu J, Ge S, Chen C, Li S, Wu X, et al. Saikosaponin A inhibits breast cancer by regulating Th1/Th2 balance. Front Pharmacol. (2019) 10:624. doi: 10.3389/fphar.2019.00624

34. Viallard JF, Pellegrin JL, Ranchin V, Schaeverbeke T, Dehais J, Longy-Boursier M, et al. Th1 (IL-2, interferon-gamma (IFN-gamma)) and Th2 (IL-10, IL4) cytokine production by peripheral blood mononuclear cells (PBMC) from patients with systemic lupus erythematosus (SLE). Clin Exp Immunol. (1999) 115:189-95. doi: 10.1046/j.1365-2249.1999.00766.x

35. Del Prete G. Human Th1 and Th2 lymphocytes: their role in the pathophysiology of atopy. Allergy. (1992) 47:4505. doi: 10.1111/j.1398-9995.1992.tb00662.x

36. Koch KC, Ye K, Clark BD, Dinarello CA. Interleukin 4 (IL) 4 up-regulates gene and surface IL 1 receptor type I in murine T helper type 2 cells. Eur J Immunol. (1992) 22:153-7. doi: 10.1002/eji.1830220123

37. Disis ML, Bernhard H, Jaffee EM. Use of tumour-responsive T cells as cancer treatment. Lancet. (2009) 373:673-83. doi: 10.1016/s0140-6736(09)60404-9

38. Maimela NR, Liu S, Zhang Y. Fates of CD8+ $\mathrm{T}$ cells in tumor microenvironment. Comput Struct Biotechnol J. (2019) 17:1-13. doi: 10.1016/j.csbj.2018.11.004

39. Kaimala S, Al-Sbiei A, Cabral-Marques O, Fernandez-Cabezudo MJ, AlRamadi BK. Attenuated bacteria as immunotherapeutic tools for cancer treatment. Front Oncol. (2018) 8:136. doi: 10.3389/fonc.2018.00136

40. Linnebacher M, Maletzki C, Klier U, Klar E. Bacterial immunotherapy of gastrointestinal tumors. Langenbecks Arch Surg. (2012) 397:557-68. doi: 10.1007/s00423-011-0892-6

41. Floros T, Tarhini AA. Anticancer cytokines: biology and clinical effects of interferon-alpha2, interleukin (IL)-2, IL-15, IL-21, and IL-12. Semin Oncol. (2015) 42:539-48. doi: 10.1053/j.seminoncol.2015.05.015

42. Waldmann TA. Cytokines in cancer immunotherapy. Cold Spring Harb Perspect Biol. (2018) 10:28472. doi: 10.1101/cshperspect.a028472

Conflict of Interest: The authors declare that the research was conducted in the absence of any commercial or financial relationships that could be construed as a potential conflict of interest.

Copyright (C) 2020 Alson, Schuyler, Yan, Samimuthu and Qiu. This is an open-access article distributed under the terms of the Creative Commons Attribution License (CC $B Y)$. The use, distribution or reproduction in other forums is permitted, provided the original author(s) and the copyright owner(s) are credited and that the original publication in this journal is cited, in accordance with accepted academic practice. No use, distribution or reproduction is permitted which does not comply with these terms. 